\title{
Competing risks to breast cancer mortality in Catalonia
} Ester Vilaprinyo*1, Rosa Gispert², Montserrat Martínez-Alonso3, Misericòrdia Carles ${ }^{4}$, Roger Pla ${ }^{5}$, Josep-Alfons Espinàs ${ }^{6}$ and Montserrat Rué ${ }^{3}$

Address: ${ }^{1}$ Institut d'Investigació Biomèdica de Bellvitge, IDIBELL, Hospitalet de Llobregat Catalonia, Spain, ${ }^{2}$ Servei d'Informació i Estudis, Departament de Salut, Generalitat de Catalunya, Catalonia, Spain, ${ }^{3}$ Institut de Recerca Biomèdica de Lleida (IRBLLEIDA)-Universitat de Lleida, Catalonia, Spain, ${ }^{4}$ Facultat de Ciències Econòmiques i Empresarials, Universitat Rovira i Virgili, Catalonia, Spain, ${ }^{5}$ Institut Català de la Salut a Terres de l'Ebre, Tortosa, Catalonia, Spain and 'Pla Director d'Oncologia, Departament de Salut, Generalitat de Catalunya, Catalonia, Spain

Email: Ester Vilaprinyo* - evilaprinyo@cmb.udl.cat; Rosa Gispert - rosa.gispert@gencat.cat; Montserrat Martínez-

Alonso - montserrat.martinez@cmb.udl.cat; Misericòrdia Carles - misericordia.carles@urv.cat; Roger Pla - rpla.ebre.ics@gencat.cat; Josep-

Alfons Espinàs - ja.espinas@iconcologia.net; Montserrat Rué - montse.rue@cmb.udl.cat

* Corresponding author

Published: 12 November 2008

BMC Cancer 2008, 8:33I doi:|0.|I86/|47|-2407-8-33|
Received: 16 January 2008

Accepted: 12 November 2008

This article is available from: http://www.biomedcentral.com//47I-2407/8/33।

(c) 2008 Vilaprinyo et al; licensee BioMed Central Ltd.

This is an Open Access article distributed under the terms of the Creative Commons Attribution License (http://creativecommons.org/licenses/by/2.0), which permits unrestricted use, distribution, and reproduction in any medium, provided the original work is properly cited.

\begin{abstract}
Background: Breast cancer mortality has experienced important changes over the last century. Breast cancer occurs in the presence of other competing risks which can influence breast cancer incidence and mortality trends. The aim of the present work is: I) to assess the impact of breast cancer deaths among mortality from all causes in Catalonia (Spain), by age and birth cohort and 2) to estimate the risk of death from other causes than breast cancer, one of the inputs needed to model breast cancer mortality reduction due to screening or therapeutic interventions.
\end{abstract}

Methods: The multi-decrement life table methodology was used. First, all-cause mortality probabilities were obtained by age and cohort. Then mortality probability for breast cancer was subtracted from the all-cause mortality probabilities to obtain cohort life tables for causes other than breast cancer. These life tables, on one hand, provide an estimate of the risk of dying from competing risks, and on the other hand, permit to assess the impact of breast cancer deaths on allcause mortality using the ratio of the probability of death for causes other than breast cancer by the all-cause probability of death.

Results: There was an increasing impact of breast cancer on mortality in the first part of the $20^{\text {th }}$ century, with a peak for cohorts born in 1945-54 in the 40-49 age groups (for which approximately $24 \%$ of mortality was due to breast cancer). Even though for cohorts born after 1955 there was only information for women under 50 , it is also important to note that the impact of breast cancer on all-cause mortality decreased for those cohorts.

Conclusion: We have quantified the effect of removing breast cancer mortality in different age groups and birth cohorts. Our results are consistent with US findings. We also have obtained an estimate of the risk of dying from competing-causes mortality, which will be used in the assessment of the effect of mammography screening on breast cancer mortality in Catalonia. 


\section{Background}

Breast cancer is the leading cause of mortality among middle-aged women in many developed countries, including Catalonia, a region in the northeast of Spain, where it accounts for a fifth of all female cancer deaths and, on the average, fourteen years of potential life lost per death from this cause [1].

Causes of death other than breast cancer may influence mortality trends in two ways: 1) by changing the number of women at risk of having breast cancer and, 2) by changing the risk of dying of breast cancer once it has developed. For example, infectious diseases at the beginning of the $20^{\text {th }}$ century reduced the number of women at risk for breast cancer and also competed with breast cancer as a cause of death in women with this disease. Thus, the effect of other causes of death in breast cancer incidence and mortality has changed over time depending on trends in other competing risks.

Rosenberg [2] used the multi-decrement life table methodology to partition overall mortality into mortality due to breast cancer and mortality due to other causes. In addition, multi-decrement life tables permit assessment of the impact that breast cancer mortality has on overall mortality by birth cohort and age. For instance, in the US, the reduction in overall mortality when removing breast cancer as a cause of death could be as high as $15 \%$ at some ages.

Evaluation of the impact of mammography and adjuvant therapy on breast cancer mortality reduction can be done using statistical models, for example those found in several studies sponsored by CISNET [3]. The modeling process requires information such as the dissemination of mammography and adjuvant treatment programs, diagnostic characteristics of mammography, breast cancer incidence and mortality. Another required input is the competing-cause mortality, taking into account that deaths due to breast cancer occur in the presence of other causes of death, the above-mentioned competing risks.
The aims of the present work are: 1) to assess the impact of breast cancer mortality on overall mortality by birth cohort and age in Catalonia in the $20^{\text {th }}$ century and 2) to assess the risk of death from other causes than breast cancer in cohorts born from 1900 to 2004. Our analysis is based on Rosenberg's work for the US and is part of a wider project that aims to model the impact of mammography in Catalonia (Spain) based on the methodology developed by Lee and Zelen for the CISNET project [4].

\section{Methods \\ Data Sources}

Catalonia is an autonomous region of Spain which has had authority over health-care planning, administration and provision since 1985 . It has approximately one sixth of the Spanish population. The Catalan Health Department has implemented an independent information system and preventive programs in the region. Population breast cancer screening programs were initiated in the early nineties [5].

By the year 2007, the Catalan Health Service was providing services to 7 million inhabitants, including 3.5 million women. The female population grew from 800.000 women in 1900 to more than 3 million in 2004. The number of breast cancer deaths increased from an average of 612 per year in the period $1975-79$ to 1060 per year in the period 1990-94 and then decreased to an average of 1000 per year in the period 2000-04. For the same periods, the percentage of breast cancer deaths among overall mortality in women increased from $3.5 \%$ to $5.2 \%$ and then decreased to $3.6 \%$.

Table 1 shows the data sources used in this study and describes their characteristics. The National Institute of Statistics (INE [6]) and the Catalan Institute of Statistics (IDESCAT [7]) have provided estimations of annual population changes since 1970. These changes were estimated by the component method [8] which takes into account vital statistics (births and deaths) and immigration to adjust official post-census estimates. We obtained the

Table I: Description and sources of data.

\begin{tabular}{|c|c|c|c|}
\hline Data & Period & Description & Source \\
\hline \multirow{3}{*}{ Population } & $1900-1970$ & Official census data (every 10 years) & National Institute of Statistics (INE) \\
\hline & $1970-1985$ & Annual population estimated by the components method* & INE \\
\hline & $1986-2004$ & Annual population estimated by the components method* & Catalan Institute of Statistics (IDESCAT) \\
\hline \multirow[t]{2}{*}{ All-cause mortality } & $1900-1974$ & Annual counts & INE \\
\hline & $1975-2004$ & Annual counts & Catalan Mortality Registry \\
\hline Breast cancer mortality & 1975-2004 & Annual counts & Catalan Mortality Registry \\
\hline
\end{tabular}

*For a detailed description of the component method see reference [8] 
mid-year population data using linear interpolation. Mortality data has been officially collected and registered by the Catalan Mortality Registry [9] and the INE. These two institutions had this responsibility during different periods (see Table 1). Population and deaths correspond to residents in Catalonia. All data are public and available from their annual reports and web pages $[6,7,9]$.

We grouped cohorts and ages into five-year categories in order to smooth data and minimize fluctuations present in some census and mortality data prior to 1960 [10], and to get more reliable estimates for young age groups. We used linear interpolation to estimate missing data or to split categories that were larger than five years in the original data source.

\section{Statistical analysis}

Deaths by calendar year were converted into deaths by year of birth (cohorts) using the relation year of birth $=$ calendar year of death - age at death.

Actuarial life table methods were used to obtain the life table functions: the age specific death rate ${ }_{n} M_{x, B Y}$, the conditional probabilities of death ${ }_{n} q_{x, B Y}$, and the proportion of expected survivors by age $l_{x, B Y}[11]$. These functions were estimated for a) the overall population, and b) for a population in which breast cancer had been eliminated as a cause of death, in the following steps:

1) We obtained age-specific death rates, ${ }_{n} M_{x, B Y}$, for age groups $[x, x+n)$ and birth cohorts $B Y$, using the expression:

$$
{ }_{n} M_{x, B Y}=\frac{{ }_{n} D_{x, B Y}}{{ }_{n} K_{x, B Y}}
$$

where ${ }_{n} D_{x, B Y}$ is the observed number of deaths and ${ }_{n} K_{x, B Y}$ the midyear population in age group $x$ to $x+n$. In our analysis $n$ is equal to 5 years.

2) We estimated overall probabilities of death ${ }_{n} q_{x, B Y}$ in the interval $[x, x+n)$ with the formula:

$$
{ }_{n} q_{x, B Y}=\frac{n M_{x, B Y}}{\frac{1}{n}\left[1+n\left(1-{ }_{n} f_{X}\right)_{n} M_{x, B Y}\right]}
$$

where ${ }_{n} f_{x}$ is the estimated fraction of years lived in the interval. Assuming a uniform distribution of deaths over time we have taken ${ }_{n} f_{x}=0.5$ for all age groups except the $0-4$ age group, where ${ }_{n} f_{0}=0.4$. All-cause probabilities of death are labeled as ${ }_{n} q_{x, B Y}^{\tau}$.
3) We estimated probabilities of death from breast cancer, $q_{x, B Y}^{b c}$, in the interval $[x, x+n)$ as described in Step 2, using only breast cancer deaths in the numerator.

4) Probabilities of death in the last age group, $85+$, were estimated as ${ }_{\infty} q_{85, B Y}^{\tau}=1$ for overall mortality, and $\infty q_{85, B Y}^{b c}={ }_{\infty} D_{85, B Y}^{b c} /_{\infty} K_{85, B Y}$ for breast cancer mortality.

5) We estimated the missing breast cancer mortality probabilities for earlier years of birth using an age-cohort model. The observed breast cancer death rates were used to fit the following quasi-Poisson model that provides maximum likelihood estimates of mortality rates $\left({ }_{n} M_{x, B Y}\right)$ based on age $(x)$ and year of birth $(B Y)$ :

${ }_{n} M_{x, B Y}=e^{\left(\beta_{0}+\beta_{\text {age } 1} x+\beta_{\text {age } 2} x^{2}+\beta_{\text {age } 3} x^{3}+\beta_{\text {age } 4} x^{4}+\beta_{\text {cohort } 1} B Y+\beta_{\text {cohort } 2} B Y^{2}\right)}$

The age-cohort model parameters estimated using the generalized linear models package of the $\mathrm{R}$ software [12] were: $\beta_{0}:-1.59 \times 10^{3}, \beta_{\text {age } 1}: 1.05, \beta_{\text {age } 2}:-2.06 \times 10^{-2}, \beta_{\text {age3 }}$ : $1.75 \times 10^{-4}, \beta_{\text {age }}:-5.21 \times 10^{-7}, \beta_{\text {cohort1 }}: 1.62$, and $\beta_{\text {cohort } 2}:$ $4.2 \times 10^{-4}$. Then the probabilities were estimated as described in Step 2. Figure 1 shows the periods and age groups where breast cancer mortality data was available (in black) or estimated (in gray).

6) We subtracted the probabilities of dying from breast cancer from the overall probabilities of death to obtain the probabilities of dying from causes other than breast cancer ${ }_{n} q_{x, B Y}^{-b c}$.

$$
{ }_{n} q_{x, B Y}^{-b c}={ }_{n} q_{x, B Y}^{\tau}-{ }_{n} q_{x, B Y}^{b c}
$$

7) We obtained the life table function $l_{x, B Y}$, which indicates the proportion of survivors at age $x$ from birth cohort $B Y$, using the expression:

$$
l_{x+n, B Y}=l_{x, B Y}\left(1-{ }_{n} q_{x, B Y}\right), \text { starting from } l_{0, B Y}=1
$$

Following the same nomenclature used in probabilities of death, the functions $l_{x, B Y}^{\tau}$ and $l_{x, B Y}^{-b c}$ were computed. The $l_{x, B Y}^{-b c}$ indicates the proportion of survivors from birth cohort $B Y$, at the beginning of each age interval, after removing breast cancer as a cause of death. 


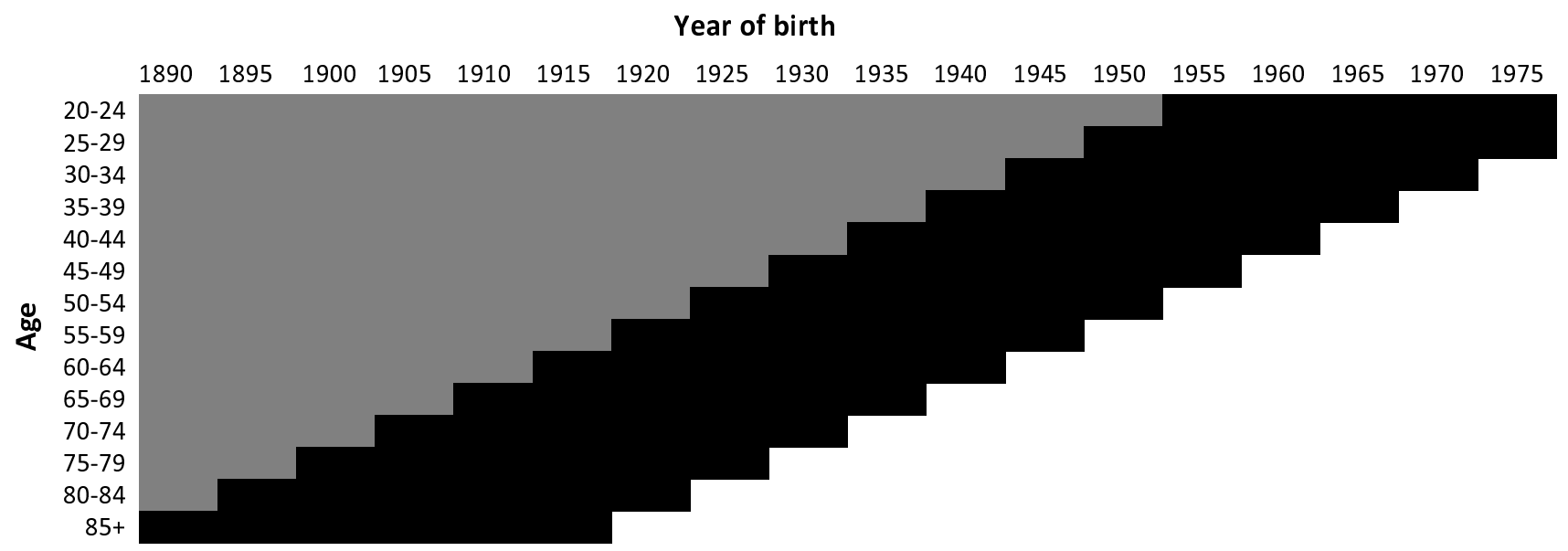

Figure I

Breast cancer probabilities of death by age and year of birth, Catalonia (Spain). The black cells indicate the available data. The grey cells indicate data that are estimated using an age-cohort model. Years of birth are grouped in five-year intervals and are labeled with the first year of the interval.

8) We computed the ratio of ${ }_{n} q_{x, B Y}^{-b c} /{ }_{n} q_{x, B Y}^{\tau}$, which measures the impact in all-cause mortality of removing deaths from breast cancer at different birth cohorts and ages. A ratio equal to 1 , in a specific group, would indicate that there is no breast cancer mortality in that group. A ratio equal to 0 would indicate that all mortality is due to breast cancer.

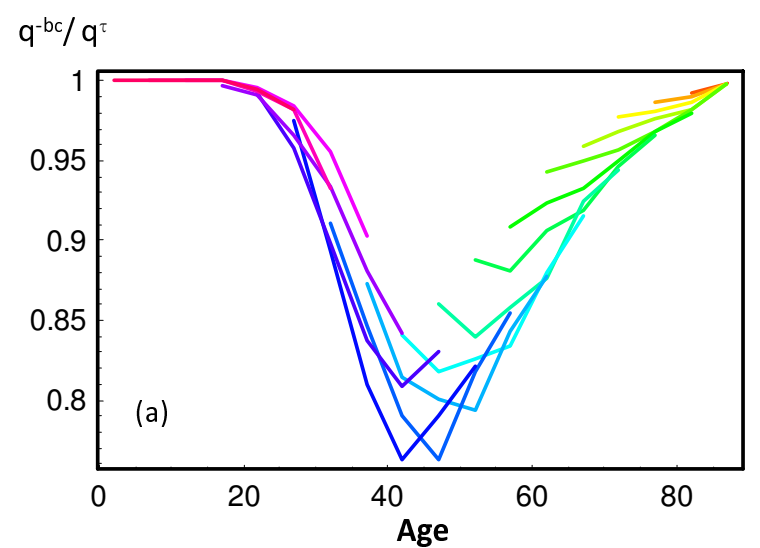

\section{Results}

Breast cancer impact on overall mortality

Cohort multi-decrement life table functions $q_{x, B Y}^{-b c}$ and $l_{x, B Y}^{-b c}$ were obtained for a population of women born between 1900 and 2004, with breast cancer removed as a cause of death.

Figure 2 presents the ratio ${ }_{n} q_{x, B Y}^{-b c} /{ }_{n} q_{x, B Y}^{\tau}$ by age and cohort of birth. This ratio can be interpreted as the proportion of deaths attributable to causes other than breast can-

\section{Figure 2}

Ratio of the probability of death with breast cancer removed to the probability of all-cause mortality $\left(q_{x}^{-b c} / q_{x}^{\tau}\right)$, by age and year of birth. (a) and (b) show results for available and for estimated data, respectively. 
cer. It facilitates evaluating the impact of removing breast cancer as a cause of death. Figures $2 \mathrm{a}$ and $2 \mathrm{~b}$ show the ratio before and after the extrapolation of breast cancer probabilities of death, respectively.

The impact of removing breast cancer as a cause of death is small under age 30 and over age 70 . Below age 30 the number of breast cancer cases is small and above age 70 mortality from other causes, such as cardiovascular diseases, increases. The largest impact of removing breast cancer as a cause of death in overall mortality is observed at ages 40-54. For specific birth cohorts, the largest observed impact was in the 40-44 age group for cohorts born in 1945-49 and the 45-49 age group for cohorts born in 1950-54 (24\% of all-cause mortality for both cohorts). Impacts assessed using observed data for cohorts born after 1955 tended to decrease. This finding is further supported by Figure 3, which shows the trend of breast cancer mortality by age for five year periods from 197579 to 2000-04. Breast cancer probability of death increased in all age groups from 1975-79 to 1990-94. In contrast, the last two studied periods, 1995-99 and 200004, show a reduction in breast cancer risk of death. Furthermore, Figure 4 shows how breast cancer mortality probabilities increased until the early 1990s, and then began to decrease, in all age groups.

\section{Risk of death from other causes than breast cancer}

The $q_{x, B Y}^{-b c}$ function estimates the risk of death from other causes when breast cancer is eliminated. The $l_{x, B Y}$ function denotes the expected number of survivors at age $x$. The $l_{x, B Y}^{-b c}$ values can be used as an estimation of the propor-

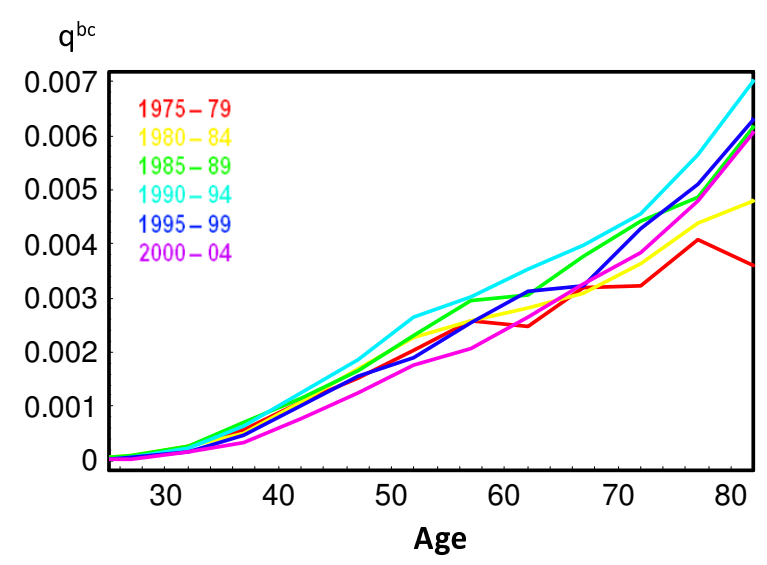

Figure 3

Probabilities of breast cancer death $\left(q_{x}^{b c}\right)$ for selected calendar years.

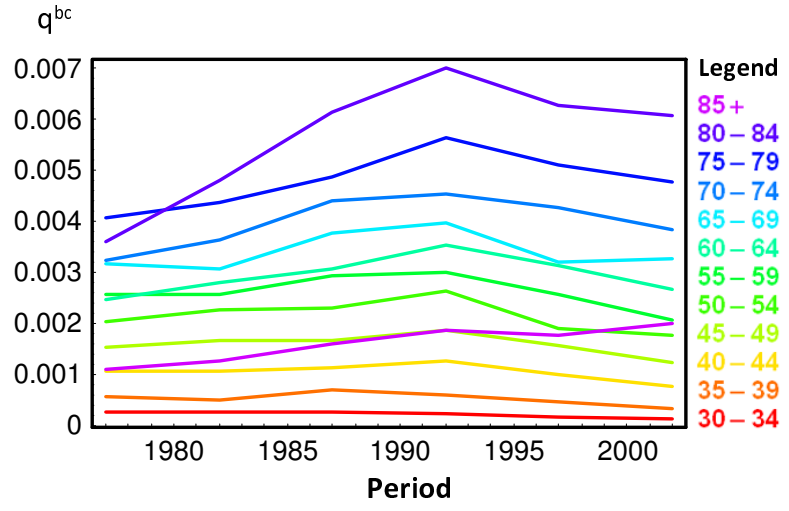

\section{Figure 4}

\section{Probabilities of breast cancer death $\left(q_{x}^{b c}\right)$ by age.}

tion of survivors, up to age $x$, if breast cancer was eliminated as a cause of death. As an illustrative example, Table 2 shows the values of $l_{x}^{\tau}$ and $l_{x, B Y}^{-b c}$ for cohorts born in the years 1920-24, 1930-34, and 1940-44. As expected, the $l_{x}^{\tau}$ data show an increasing trend in the probability of surviving to a specific age by birth cohort. For instance, the proportion of survivors at age 20 was $75 \%$ for women born in the early 1920s, and increased to $90 \%$ for women born in the early 1940s.

The column Dif. in Table 2 contains the difference $l_{x, B Y}^{-b c}-l_{x, B Y}^{\tau}$ per 10,000 women. It can be interpreted as the number of women saved up to age $x$ from an initial cohort of 10,000 women, when breast cancer is removed as a cause of death. If there were no breast cancer deaths, for each 10,000 women born in 1920-24 approximately 93 would have been saved before arriving at age 70, and 118 women for the cohort born in 1930-34.

Figure 2 and Table 2 show two different approaches to evaluating trends in breast cancer mortality in the presence of other causes of death by age and cohort. The $q_{x, B Y}^{-b c}$ estimates competing-cause mortality, the ratio ${ }_{n} q_{x, B Y}^{-b c} /{ }_{n} q_{x, B Y}^{\tau}$ shows the impact of breast cancer over global mortality, and $l_{x, B Y}^{\tau}-l_{x, B Y}^{-b c}$ indicates the number of women saved up to age $x$ if breast cancer was removed as a cause of death.

\section{Discussion and conclusion}

Our study shows that breast cancer mortality in Catalonia has experienced important changes over the last century. 
Table 2: Proportion of survivors, $l_{x}^{\tau}$, and proportion of survivors after removing breast cancer as a cause of death, $l_{x}^{-b c}$, by age $\mathrm{x}$ and cohort of birth.

\begin{tabular}{|c|c|c|c|c|c|c|c|c|c|}
\hline \multirow[t]{3}{*}{ Ages } & \multicolumn{9}{|c|}{ Year of Birth } \\
\hline & \multicolumn{3}{|c|}{$1920-24$} & \multicolumn{3}{|c|}{ 1930-34 } & \multicolumn{3}{|c|}{$1940-44$} \\
\hline & $l_{x}^{\tau}$ & $l_{x}^{-b c}$ & Dif.* & $l_{x}^{\tau}$ & $l_{x}^{-b c}$ & Dif.* & $l_{x}^{\tau}$ & $l_{x}^{-b c}$ & Dif.* \\
\hline $20-24$ & 0.7474 & 0.7474 & 0 & 0.8499 & 0.8499 & 0 & 0.8977 & 0.8977 & 0 \\
\hline $25-29$ & 0.7338 & 0.7338 & 0.14 & 0.8435 & 0.8435 & 0.17 & 0.8953 & 0.8953 & 0.17 \\
\hline $30-34$ & 0.7229 & 0.7230 & 0.83 & 0.8389 & 0.8390 & 0.97 & 0.8927 & 0.8928 & 0.97 \\
\hline $35-39$ & 0.7162 & 0.7165 & 3.00 & 0.8346 & 0.8350 & 3.56 & 0.8894 & 0.8897 & 3.20 \\
\hline $40-44$ & 0.7096 & 0.7104 & 7.91 & 0.8288 & 0.8297 & 9.42 & 0.8854 & 0.8862 & 8.20 \\
\hline $45-49$ & 0.7017 & 0.7034 & 16.45 & 0.8218 & 0.8236 & 17.74 & 0.8803 & 0.8821 & 17.65 \\
\hline $50-54$ & 0.6913 & 0.6941 & 28.61 & 0.8129 & 0.8159 & 30.16 & 0.8730 & 0.8762 & 32.23 \\
\hline $55-59$ & 0.6760 & 0.6800 & 39.65 & 0.8016 & 0.8064 & 48.20 & 0.8620 & 0.8675 & 54.90 \\
\hline $60-64$ & 0.6574 & 0.6630 & 56.01 & 0.7853 & 0.7924 & 70.91 & $0.848 I$ & 0.8557 & 76.17 \\
\hline $65-69$ & 0.6342 & 0.6414 & 72.63 & 0.7634 & 0.7731 & 96.93 & - & - & - \\
\hline $70-74$ & 0.6001 & 0.6094 & 93.00 & 0.7320 & 0.7438 & 117.75 & - & - & - \\
\hline $75-79$ & 0.5499 & 0.5612 & 112.95 & - & - & - & - & - & - \\
\hline $80-84$ & 0.4733 & 0.4859 & 125.85 & - & - & - & - & - & - \\
\hline $85+$ & - & - & - & - & - & - & - & - & - \\
\hline
\end{tabular}

Note: $l_{x}^{-b c}$ can be interpreted as the proportion of women at risk of dying from competing risks at age $x$.

* Indicates the number of women saved up to age $x$ from an initial cohort of 10,000 women, if breast cancer was removed as a cause of death.

There was an increasing impact of breast cancer on overall mortality in the first part of the century, with a peak for cohorts born in 1945-54 in the 40-49 age groups (approximately 24\% of mortality was due to breast cancer in these age groups and cohorts). This increasing impact could be explained by a decrease in mortality from other causes of death and increased breast cancer incidence and mortality due to changes in lifestyle and reproductive patterns [13]. In Catalonia, mortality from all causes in women has been decreasing an average of $1.6 \%$ per year since 1978 [14], whereas breast cancer incidence increased 2.2\% per year between 1980-97 [15].

The subsequent decrease in impact was due, primarily, to the observed reduction in mortality from breast cancer during the 1990s, which affected all age groups (see [16] and official reports by the Catalan Department of Health [14]). Furthermore, the significant reduction in recent decades implies important gains in life expectancy for middle-aged women [17]. This reduction has been attributed to the use of mammography and adjuvant treatments in the US [18]. The phenomenon is now being studied in Catalonia.

In Catalonia, as in the US, breast cancer mortality had the greatest impact on global mortality in the 40-54 age groups during the $20^{\text {th }}$ century [2]. On one hand, breast cancer incidence and mortality are low before the age of
40 [19]. Although breast cancer incidence and mortality increase with age, after age 50 there are other causes of death (competing risks) that are acting simultaneously, and therefore breast cancer may have less impact on overall mortality.

It is worthwhile to note that the values of the ratio ${ }_{n} q_{x, B Y}^{-b c} /{ }_{n} q_{x, B Y}^{\tau}$ were higher in the US, which means that the impact of breast cancer mortality was lower. The ratio ${ }_{n} q_{x, B Y}^{-b c} /{ }_{n} q_{x, B Y}^{\tau}$ depends on two risks, the risk of dying of causes other than breast cancer (numerator) and the risk of dying of any cause (denominator). For cohorts of women born in the middle of the $20^{\text {th }}$ century, overall mortality rates at ages 40 to 54 years were $40 \%$ to $60 \%$ higher in the US than in Catalonia [20]. On the other hand, breast cancer mortality rates for these ages and cohorts were similar or slightly higher in Spain than the US. Since breast cancer mortality rates represent about one fifth of the mortality in these age groups, differences in the overall mortality risk in the US and Catalonia may explain the differences seen in the ${ }_{n} q_{x, B Y}^{-b c} /{ }_{n} q_{x, B Y}^{\tau}$ ratio. But, other factors like reproductive patterns, hormonal replacement therapy use and fat intake could explain dif- 
ferences in the impact of breast cancer mortality in both countries.

Results from Rosenberg show that the lowest ${ }_{n} q_{x, B Y}^{-b c} /{ }_{n} q_{x, B Y}^{\tau}$ ratio (the highest impact of breast cancer mortality) for women born in 1930 occurs at older ages than for those born in 1950. This pattern is also shown in our data before any extrapolation (Figure 2a). In Catalonia this is related with a clear period effect during the 1990s. As we show in Figures 3 and 4, trends for breast cancer death probabilities by age increased from the 1970 s to the 1990s and started a decreasing trend after that for all age groups.

The declines in mortality from breast cancer, which began in Europe in the late 1980s may be attributed in part to earlier detection by screening programs. But, since the declining trends started before screening was introduced and occurred also in non-screened age groups, improved cancer treatments such as adjuvant chemotherapy and tamoxifen may have been important determinants of breast cancer mortality reduction [21]. In Catalonia, breast cancer mortality started to decrease at the beginning of the 1990s, concurrent with the dissemination of mammography. Since the effect of screening would be seen some years later, treatments probably had an important role during the 1990s. The contribution of each of these factors still needs to be determined in Catalonia.

Our work was undertaken in the Catalan region and not in the whole country of Spain. This is due to the fact that there is no national cancer registry in Spain. Instead, there are 12 local cancer registries, which show differences in the incidence of breast cancer by region (probably due to different reproductive patterns and degrees of economic development). The only statistics that are collected in a standardized manner at the national level are mortality data. Also, this study is part of a project that aims to assessing the cost-effectiveness of different early detection strategies on the reduction of breast cancer mortality. The costeffectiveness analysis needs information on outcomes and costs that would be difficult to obtain at the national level. On the other hand, since Catalonia has approximately one sixth of the Spanish population, we believe that some of the results of our studies will be relevant for all of Spain.

This study has several limitations. First, the multi-decrement method that we used assumes independence of causes of death, which means that when breast cancer mortality is removed, the risk of dying due to the remaining causes of death is not affected. This assumption may not be true, since different causes of death may share the same risk factors. For example, body weight, smoking, and diet are associated not only with breast cancer but also with other health problems, like cardiovascular diseases. Therefore, the probability of dying of other causes could change when breast cancer death is removed. In our study, if mortality from other causes had decreased when eliminating breast cancer mortality, the impact of breast cancer mortality would be higher than the reported values. There are methods for competing risks analysis, such as cumulative incidence functions, that do not make any assumptions about independence of risks, but need information on the relationships among them $[22,23]$. These methods are more complex than multi-decrement life tables and have been used to evaluate the effects of explanatory variables, such as assessing the effects of therapy in different groups with multiple endpoints. On the other hand, Chin Long Chiang proposed a method [24] that takes into account different conditional probabilities of death (crude, net and partial crude) which reflect the relationships among the different risks of death acting simultaneously. Nevertheless, although these methods could be more accurate than the one used in our work, they make assumptions about the risks' associations requiring information that was not available to us such as causes of death for women with breast cancer. Therefore, we chose the multi-decrement method, that Rosenberg also used [2], because it is simple and adequate to fulfill our objectives.

A second limitation concerns quality of information. The validity of population and mortality data has increased over time, but there may be errors in data from the earliest years of the study. We observed some fluctuations in the late 1930s and early 1940s, coinciding with the Spanish Civil War, a problem that could be addressed by smoothing the life table series' before using them in subsequent analyses. Also, to minimize variability we aggregated data in 5-year age groups and calendar years. The quality of mortality statistics has also changed over time, but different studies have shown that in Spain, deaths from cancer as a whole and leading cancer sites (lung, colon-rectum, prostate, stomach, pancreas, female breast, uterus, brain, leukemia, lymphomas and myeloma) were properly coded $[25,26]$. Furthermore, breast cancer mortality data was provided by the Catalan Mortality Registry which, since the beginning of the 1980s, collects data and codes causes of death consistently and has adequate quality standards.

A third limitation arises from the assumptions made when completing information on breast cancer mortality probabilities for years without available data. Data from available years seemed adequate to us to fit an age-cohort model that predicted missing information. Other approximations such as the assumption that the probabilities of 
dying from breast cancer at a given age would be the same in neighboring years with data, as Rosenberg did, do not change the main results (data not shown). In any case, extrapolated data for the earlier cohorts may differ from the actual values and therefore results based on these data should be interpreted with caution.

In summary, we obtained cohort life tables for causes of death other than breast cancer in Catalonia (Spain). This makes it possible to quantify the impact of removing breast cancer on overall mortality in different age groups and birth cohorts $\left({ }_{n} q_{x, B Y}^{-b c} /{ }_{n} q_{x, B Y}^{\tau}\right)$. As with the US results, our analysis found that the greatest impact of breast cancer mortality on overall mortality was for women aged $40-54$ born in the middle of the $20^{\text {th }}$ century. The multi-decrement life tables method also provided estimations of the risk of dying from competing risks that will be used in the assessment of the effect of mammography screening on breast cancer mortality.

\section{Competing interests}

The authors declare that they have no competing interests.

\section{Authors' contributions}

$\mathrm{MR}, \mathrm{EV}, \mathrm{MC}, \mathrm{RP}$ and JAE participated in design and coordination of the study. EV and MR performed the statistical analysis and drafted the manuscript. RG provided mortality data and contributed to the interpretation of results. MM developed the age-cohort model for breast cancer mortality. All authors read and approved the final manuscript.

\section{Acknowledgements}

This work has been funded by projects PI06/I649 and PI06/90355 from the Fondo de Investigación Sanitaria, Spanish Ministry of Health, and project 068/27/06 from the Agència per a l'Avaluació de la Tecnologia i la Recerca Mèdiques, Department of Health, Generalitat de Catalunya, Spain. We are indebted to the reviewers for their comments, which have improved the quality of the manuscript.

\section{References}

I. García F, Puigdefàbregas A, Gispert R: Anàlisi de la mortalitat a Catalunya, 2005. 2007 [http://www.gencat.cat $/$ salut $/$ depsalut $/ \mathrm{html} /$ ca/dir 1932/index.html]. Barcelona: Catalan Department of Health, Servei d'informació i estudis. Generalitat de Catalunya

2. Rosenberg MA: Competing risks to breast cancer mortality. J Natl Cancer Inst Monogr 2006:15-19.

3. Habbema JD, Schechter CB, Cronin KA, Clarke LD, Feuer EJ: Modeling cancer natural history, epidemiology, and control: reflections on the CISNET breast group experience. J Natl Cancer Inst Monogr 2006: I22-126.

4. Lee S, Zelen M: A stochastic model for predicting the mortality of breast cancer. J Natl Cancer Inst Monogr 2006:79-86.

5. Masuet $C$, Seculi E, Brugulat $P$, Tresserras R: The practice of preventive mammography in Catalonia [Spain]: a step forward. Gac Sanit 2004, 1 8(4):32 I-325.

6. INEbase: Official statistics website of Spain [http://
] www.ine.es/en/inebmenu/indice en.htm]
7. IDESCAT:Official statistics website of Catalonia [http:// www.idescat.net]

8. S Shryock H, Siegel JS, Associates: The Methods and Materials of Demography (Studies in Population). San Diego: Academic Press; 1976.

9. Servei d'informació i estudis: Anàlisi de la mortalitat a Catalunya, diversos anys. [http://www.gencat.cat/salut/depsalut/html/ ca/dirl932/index.html]. Barcelona: Catalan Department of Health, Generalitat de Catalunya

10. Glei DA, Redondo RG, Argüeso A, Canudas-Romo V: Description of Spanish data. In Human Mortality Database University of California, Berkeley (USA), and Max Planck Institute for Demographic Research (Germany). http://www.mortality.org or http:// www.humanmortality.de

II. Elandt-Johnson RC, Johnson NL: Survival Models and Data Analysis. John Wiley \& Sons edn. New York: John Wiley \& Son; 1980.

12. R Development Core Team: R: A language and environment for statistical computing. 2008 [http://www.R-project.org]. Vienna, Austria: R Foundation for Statistical Computing

13. Kelsey JL, Gammon MD: The epidemiology of breast cancer. CA Cancer J Clin 199I, 4I(3): I46-I65.

14. Puig X, López-Abente G, Gispert R, Freitas A, Puigdefàbregas A: Tendències de la mortalitat a Catalunya, 1978-2002. Models edat/període/cohort. 2005 [http://www.gencat.cat/salut/depsalut/ pdf/t| 978-2002.pdf]. Catalan Department of Health, Generalitat de Catalunya

15. Borràs JMBJ, Bosch FX, Fernández E, Galceran J, Gispert R, González JR, Izquierdo A, Marcos R, Moreno V, Peris M, Puig X, Sánchez V, Viladiu P: Càncer Catalunya 200 I. L'Hospitalet: Institut Català d'Oncologia, Departament de Sanitat i Seguretat Social; 2001.

16. Puig X, Ginebra J, Gispert R: Analysis of mortality time trend using generalized linear models. Gac Sanit 2005, I 9(6):48I-485.

17. Serra I, Gispert R, Puig X, Torné M, Puigdefàbregas A: Impacte de l'edat i les causes de mort en els canvis de l'esperança de vida. Catalunya, 1987-2002. 2006 [http://www.gencat.cat/salut/ depsalut/pdf/imcauses2006.pdf]. Catalan Department of Health, Generalitat de Catalunya

18. Cronin KA, Feuer EJ, Clarke LD, Plevritis SK: Impact of adjuvant therapy and mammography on U.S. mortality from 1975 to 2000: comparison of mortality results from the cisnet breast cancer base case analysis. J Natl Cancer Inst Monogr 2006: I I 2-12 I.

19. Parkin DM, Whelan SL, Ferlay J, Storm H: Cancer Incidence in Five Continents, Vol. I to VIII. In IARC CancerBase No 7, Lyon IARC CancerBase No. 7, Lyon; 2005.

20. Human Mortality Database [http://www.mortality.org/]

21. Botha JL, Bray F, Sankila R, Parkin DM: Breast cancer incidence and mortality trends in 16 European countries. Eur J Cancer 2003, 39( I 2): 1718-1729.

22. Fine J, Gray R: A Proportional Hazards Model for the Subdistribution of a Competing Risk. J Am Statist Ass 1999, 94(446):496-509.

23. Schairer C, Mink PJ, Carroll L, Devesa SS: Probabilities of death from breast cancer and other causes among female breast cancer patients. J Natl Cancer Inst 2004, 96( 17): | I I I-I32|.

24. Chiang CL: Competing risks in mortality analysis. Annu Rev Public Health 1991, I 2:28I-307.

25. Perez-Gomez B, Aragones N, Pollan M, Suarez B, Lope V, Llacer A, Lopez-Abente G: Accuracy of cancer death certificates in Spain: a summary of available information. Gac Sanit 2006, 20(Suppl 3):42-5I.

26. de Aran Bares M, Perez G, Rosell J, Molina P: Exactness of mortality statistics by external and natural causes of death with medico-legal intervention in Catalonia, 1996. Gac Sanit 2000, I4(5):356-362.

\section{Pre-publication history}

The pre-publication history for this paper can be accessed here:

http://www.biomedcentral.com/1471-2407/8/331/pre $\underline{\mathrm{pub}}$ 